\title{
The expression and clinical significance of PI3K, pAkt and VEGF in colon cancer
}

\author{
YINXU ZHANG ${ }^{1,2}$, XIAOMEI LIU ${ }^{2}$, JUNHUA ZHANG ${ }^{2}$, LEIYU LI $^{2}$ and CHUNYING LIU ${ }^{1}$ \\ ${ }^{1}$ Department of Pathology, Liaoning University of Traditional Chinese Medicine, Shenyang 110032; \\ ${ }^{2}$ Department of Surgery, The First Affiliated Hospital of Liaoning Medical University, Jinzhou, Liaoning 121001, P.R. China
}

Received March 28, 2012; Accepted July 10, 2012

DOI: $10.3892 / \mathrm{ol} .2012 .822$

\begin{abstract}
Background PI3K/Akt signaling has been shown to be activated in a variety of cancers. However, the correlation between Akt activation and VEGF expression is unclear in colon cancer tissues. This study aimed to investigate the expression and predictive value of phosphorylated Akt (pAkt) and VEGF in colon cancer tissues. The expression of PI3K, pAkt and VEGF was detected by immunohistochemical staining in 60 samples of colon cancer tissues and their corresponding adjacent normal colon tissues. In addition, the correlations between the expression levels of the 3 proteins and the clinicopathological parameters of the colon cancer cases were analyzed. The positive rates of PI3K, pAkt and VEGF expression were $71.7 \%(43 / 60), 68.3 \%(41 / 60)$ and $61.7 \%(37 / 60)$ in colon cancer, respectively, which were significantly higher than in adjacent normal colon tissues $(\mathrm{P}<0.001)$. Correlation analyses showed that PI3K expression was not significantly associated with the gender or age of the patients, tumor size or differentiation $(\mathrm{P}>0.05)$, but was closely associated with serous coat infiltration and lymphatic metastasis of colon cancer $(\mathrm{P}<0.05)$. Neither pAkt nor VEGF expression were significantly associated with the gender or age of the patients, or tumor differentiation $(\mathrm{P}>0.05)$, but were closely associated with tumor size, serous coat infiltration and lymphatic metastasis of colon cancer $(\mathrm{P}<0.05)$. In addition, the expression levels of $\mathrm{Pl} 3 \mathrm{~K}$ and pAkt were positively correlated with that of VEGF in colon cancer tissues $(\mathrm{P}<0.05)$. Our data show a positive correlation between $\mathrm{PI} 3 \mathrm{~K} / \mathrm{Akt}$ activation and VEGF expression in colon cancer tissues and indicate that pAkt is an independent prognostic marker for colon cancer patients.
\end{abstract}

Correspondence to: Professor Chunying Liu, Department of Pathology, No.79 Chongshan Road, Liaoning University of Traditional Chinese Medicine, Huanggu District, Shenyang 110032, P.R. China

E-mail: chunying99@163.com

Key words: colon cancer, PI3K/Akt, VEGF, angiogenesis, prognosis

\section{Introduction}

Colon cancer is the most common malignancy and the third leading cause of cancer-related mortality in China. Recently, the incidence and mortality of colon cancer have increased gradually in China (1). Therefore, a deeper understanding of the molecular pathology of colon cancer is critical for establishing novel therapeutic and diagnostic strategies against this potentially lethal disease.

The PI3K/Akt pathway is crucially involved in mammalian cell survival and resistance to apoptosis (2). PI3K/Akt signaling has been shown to be overactivated in a variety of cancers, thereby promoting cancer initiation and progression (3). However, angiogenesis plays a significant role in tumor metastasis and vascular endothelial growth factor (VEGF) is one of the most effective factors that promotes tumor neovessel angiogenesis. The high expression level of VEGF has been shown to be correlated with the metastasis of several cancers, including esophageal and pancreatic cancer $(4,5)$. However, few studies have examined the correlation between the activation of PI3K/Akt signaling and VEGF expression in colon cancer tissue samples. Therefore, in the present study we mainly employed immunohistochemical methods to detect the protein expression of PI3K, Akt and VEGF in colon cancer tissues and then analyzed the correlations of the expression levels of these proteins with the clinicopathological features of colon cancer.

\section{Materials and methods}

Sample collection and case data. From April 1990 to February 2007, samples from 60 cases of colon cancer were collected from the Department of Surgery at the First Affiliated Hospital of Liaoning Medical University, Jinzhou, China. The clinical samples were obtained from surgically removed, pathologically confirmed colon cancer. The patients included 34 males and 26 females and their ages ranged from 39 to 74 years (median age, 53.6 years). None of the patients accepted preoperative radiotherapy or chemotherapy. Among the 60 patients, 4 succumbed to the disease and the remaining 56 patients were followed up for 10 to 204 months following surgery. The pathological classifications of colon cancer were based on tumor size, infiltration, differentiation and lymph node metastasis. Normal colon tissue samples adjacent to the colon cancer were 
Table I. Expression of PI3K, pAkt and VEGF in colon cancer and normal colon tissues.

\begin{tabular}{lcccccc}
\hline Group (n) & $\mathrm{PI} 3 \mathrm{~K}(+)$ & $\%$ & $\mathrm{pAkt}(+)$ & $\%$ & $\mathrm{VEGF}(+)$ & $\%$ \\
\hline Colon cancer (60) & 43 & 71.7 & 41 & 65 & 37 & 61.7 \\
Normal colon (20) & 9 & 45 & 7 & 35 & 6 & 30 \\
\hline
\end{tabular}

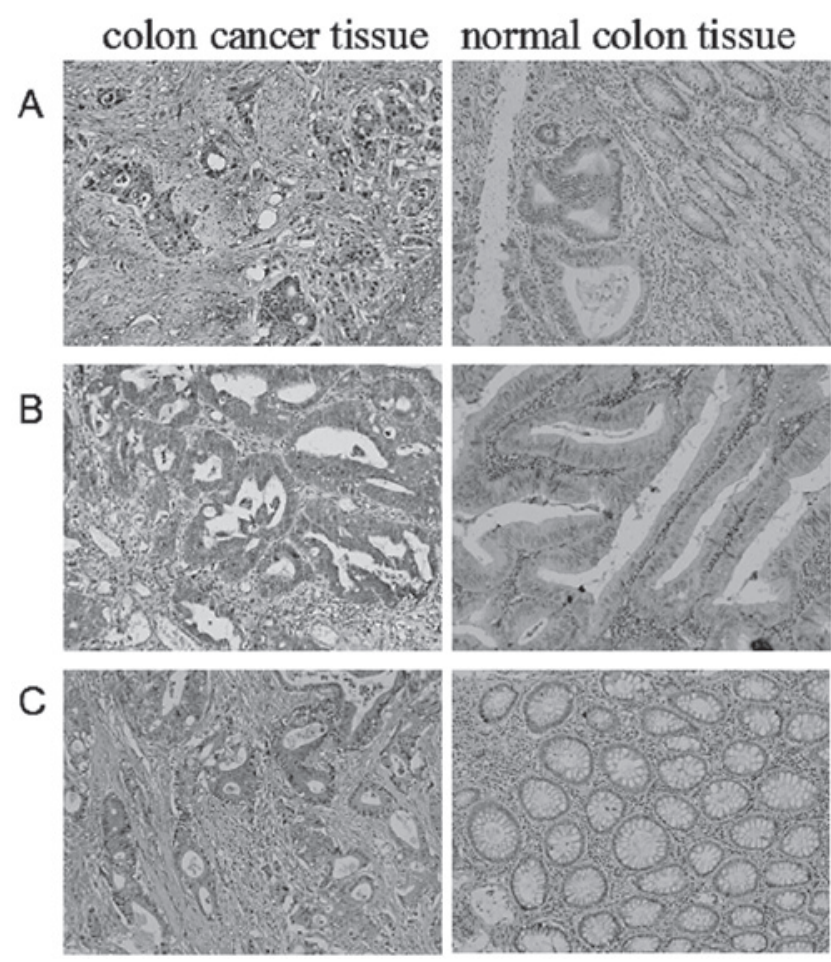

Figure 1. Representative images of immunohistochemical staining to detect the expression of PI3K, pAkt and VEGF in clinical colon tissues. (A) PI3K immunoreactivity was detected in the colon cancer tissue but not in adjacent normal colon tissue. (B) pAkt immunoreactivity was detected in the colon cancer tissue but not in adjacent normal colon tissue. (C) VEGF immunoreactivity was detected in the colon cancer tissue but was weak in adjacent normal colon tissue. Magnification, x400.

also collected from each case and used as negative controls. The collection of clinical samples was approved by the Ethics Committee of Liaoning Medical University, and all patients gave their written informed consent.

Immunohistochemical staining. Tumor tissues and normal tissues were fixed in $10 \%$ formaldehyde and were paraffin wax-embedded. Subsequently, the tissues were cut into 5-mm thick serial sections, which were washed carefully with $0.01 \mathrm{M}$ phosphate-buffered saline (PBS) three times, and then blocked with $2 \%$ goat serum in $0.01 \mathrm{M}$ PBS containing $0.3 \%$ Triton $\mathrm{X}-100$ for $1 \mathrm{~h}$ at room temperature. The sections were incubated at $4^{\circ} \mathrm{C}$ overnight with rabbit antibody against human PI3K, pAkt or VEGF (Santa Cruz Biotechnology, Inc., Santa Cruz, CA, USA). Subsequently, the slides were subjected to immunohistochemical staining using a streptavidin-biotinperoxidase SP kit (Boster, Wuhan, China). After visualizing the reaction with 3,3'-Diaminobenzidine (DAB), the slides were counterstained with haematoxylin. For negative controls, the primary antibodies were replaced with PBS.
Table II. Correlation between PI3K, pAkt and VEGF expression in colon cancer tissues.

\begin{tabular}{lrrrrrr}
\hline & \multicolumn{3}{c}{ pAkt } & & \multicolumn{2}{c}{ VEGF } \\
\cline { 2 - 3 } \cline { 5 - 6 } Group (n) & + & & - & & + & - \\
\hline PI3K(+) (43) & 34 & & 9 & & 31 & 12 \\
PI3K(-) (17) & 7 & & 10 & & 6 & 11 \\
& & & $\mathrm{P}=0.004$ & & & $\mathrm{P}=0.008$ \\
\hline
\end{tabular}

Evaluation of immunohistochemical staining. Following immunohistochemical staining, the tissue sections were evaluated independently by two blinded investigators who provided a consensus opinion of the staining patterns observed under light microscopy. Five fields were randomly selected and immunohistochemical staining of the cells was assessed according to the proportion of cells stained and the staining intensity. The proportion of cells stained was assessed using a semiquantitative 4-point scale: $0,<10 \%$ staining; 1, 10-20\% staining; 2, 21-50\% staining; and 3,>50\% staining. The staining intensity was also graded using a 4-point scale: 0 , no staining; 1, light yellow; 2, brown; and 3, dark brown. The combined score was calculated by multiplying the individual scale of the proportion and intensity (range 0-9) and was assessed as follows: $0-2$, negative staining (-); 3-6, positive staining (+); and $\geq 6$, strong positive staining $(++)$.

Statistical analysis. The differences between groups were tested for significance using the $\chi^{2}$ test. The Pearson test was performed for correlation analysis. Statistical analyses were performed using SPSS version 15.0 (SPSS Inc., Chicago, IL, USA). $\mathrm{P}<0.05$ was considered to indicate a statistically significant result.

\section{Results}

PI3K, pAkt and VEGF are highly expressed in colon cancer tissues. Immunohistochemical staining showed that the positive rates of PI3K, pAkt and VEGF expression were $71.7 \%$ (43/60), 68.3\% (41/60) and 61.7\% (37/60), respectively, among 60 colon cancer tissue samples. In the control group of adjacent normal colon tissues, the positive rates of PI3K, pAkt and VEGF expression were clearly lower than in the colon cancer group (Fig. 1). The differences between the two groups were statistically significant $(\mathrm{P}<0.05$; Table I).

Expression of PI3K, pAkt and VEGF have a positive correlation in colon cancer tissues. Among the 43 colon cancer tissues in which PI3K was highly expressed, pAkt was highly 
Table III. Correlation between PI3K, pAkt and VEGF expression in colon cancer and clinicopathological features.

\begin{tabular}{|c|c|c|c|c|c|c|c|c|c|c|}
\hline $\begin{array}{l}\text { Clinicopathological } \\
\text { feature }\end{array}$ & $\mathrm{n}$ & $\operatorname{PI} 3 \mathrm{~K}(+)$ & $\%$ & $\chi^{2} / \mathrm{P}$-value & $\operatorname{pAkt}(+)$ & $\%$ & $\chi^{2} / \mathrm{P}$-value & VEGF(+) & $\%$ & $\chi^{2} / \mathrm{P}$-value \\
\hline \multicolumn{11}{|l|}{ Gender } \\
\hline Male & 28 & 21 & 75 & $0.287 / 0.592$ & 19 & 67.9 & $0.188 / 0.664$ & 19 & 67.9 & $0.851 / 0.356$ \\
\hline Female & 32 & 22 & 68.8 & & 20 & 62.5 & & 18 & 56.3 & \\
\hline \multicolumn{11}{|l|}{ Age (years) } \\
\hline$<65$ & 46 & 34 & 73.9 & $0.490 / 0.484$ & 29 & 63 & $0.066 / 0.798$ & 26 & 56.5 & $1.373 / 0.241$ \\
\hline$\geq 65$ & 14 & 9 & 64.3 & & 10 & 71.4 & & 11 & 78.6 & \\
\hline \multicolumn{11}{|l|}{ Tumor size $(\mathrm{cm})$} \\
\hline$<5$ & 26 & 16 & 61.5 & $2.318 / 0.128$ & 13 & 50 & $4.538 / 0.033$ & 10 & 38.5 & $10.452 / 0.001$ \\
\hline$\geq 5$ & 34 & 27 & 79.4 & & 26 & 76.5 & & 27 & 79.4 & \\
\hline \multicolumn{11}{|l|}{$\begin{array}{l}\text { Serous coat } \\
\text { infiltration }\end{array}$} \\
\hline Yes & 39 & 33 & 84.6 & $11.613 / 0.001$ & 29 & 74.4 & $4.290 / 0.038$ & 28 & 71.8 & $4.835 / 0.028$ \\
\hline No & 21 & 10 & 47.6 & & 10 & 47.6 & & 9 & 42.9 & \\
\hline \multicolumn{11}{|l|}{ Differentiation } \\
\hline High/medium & 45 & 31 & 68.9 & $0.318 / 0.528$ & 26 & 57.8 & $0.178 / 0.604$ & 26 & 57.8 & $0.151 / 0.656$ \\
\hline Low & 15 & 12 & 80 & & 13 & 86.7 & & 11 & 73.3 & \\
\hline \multicolumn{11}{|l|}{$\begin{array}{l}\text { Lymphatic } \\
\text { metastasis }\end{array}$} \\
\hline Yes & 36 & 30 & 83.3 & $4.602 / 0.032$ & 28 & 77.8 & $6.459 / 0.011$ & 26 & 72.2 & $4.242 / 0.039$ \\
\hline No & 24 & 14 & 58.3 & & 11 & 45.8 & & 11 & 45.8 & \\
\hline
\end{tabular}

expressed in 32 cases and VEGF protein was highly expressed in 31 cases. Furthermore, among the 17 colon cancer tissues with negative staining for PI3K, pAkt staining was negative in 12 cases and VEGF staining was negative in 13 cases. The Pearson analysis showed that PI3K expression had a positive correlation with pAkt and VEGF expression in colon cancer tissues $(\mathrm{P}<0.05$, Table II).

Correlation between the expression of PI3K, pAkt and VEGF and clinicopathological parameters of colon cancer. To investigate the clinical significance of the expression of PI3K, pAkt and VEGF in colon cancer, we analyzed the clinicopathological parameters of the patients. Statistical analysis showed that PI3K expression was not significantly associated with the gender or age of the patients, tumor size or differentiation ( $P>0.05)$, but was closely associated with serous coat infiltration and lymphatic metastasis of colon cancer $(\mathrm{P}<0.05)$. Neither pAkt nor VEGF expression were significantly associated with the gender or age of the patients, or tumor differentiation ( $\mathrm{P}>0.05)$, but were closely associated with tumor size, serous coat infiltration and lymphatic metastasis of colon cancer $(\mathrm{P}<0.05$; Table III).

\section{Discussion}

Despite the progress in the development of effective diagnostic and therapeutic strategies for colon cancer, treatment of the disease remains a challenge due to the overall poor prognosis of colon cancer patients. Consequently, great efforts have been taken recently to identify novel prognostic and predictive biomarkers for colon cancer and recent studies reported that several biomarkers were able to predict the survival of colon cancer patients (6-8).

The PI3K/Akt pathway is involved in cellular survival by inhibiting apoptotic processes and stimulating cell growth and proliferation. The PI3K/Akt pathway is commonly activated in human cancers and has been recognized as an important target for anti-cancer therapy. In addition, the negative prognostic value of the PI3K/Akt pathway has been revealed in several types of cancer (9-11). However, the clinical significance of the activation of PI3K/Akt in colon cancer remains uncertain. VEGF is a potent angiogenic factor that promotes tumor metastasis and angiogenesis (12). A recent study demonstrated that the phosphorylation of Akt and ERK1/2 is required for VEGF-induced proliferation and migration of lymphatic endothelium, suggesting that PI3K/Akt signaling could cross-talk with VEGF signaling to promote tumor angiogenesis (13). The phosphorylation of Akt is established as a hallmark for the activation of PI3K/Akt signaling but few studies have examined the correlation between the phosphorylation of Akt and VEGF expression in colon cancer tissue samples. Therefore, in the present study we aimed to detect the protein levels of PI3K, pAkt and VEGF in colon cancer tissues and investigate the correlations of the expression levels of these proteins with the clinicopathological variables of colon cancer.

By immunohistochemical staining, we showed that the positive rates of $\mathrm{PI} 3 \mathrm{~K}$, pAkt and VEGF expression were $71.7 \%$ (43/60), 68.3\% (41/60) and 61.7\% (37/60), respectively, in colon cancer, which were significantly higher than in adjacent normal colon tissues $(\mathrm{P}<0.001)$. The correlation analysis 
showed that the expression level of PI3K was closely associated with serous coat infiltration and lymphatic metastasis of colon cancer $(\mathrm{P}<0.05)$, and the expression levels of pAkt and VEGF were closely associated with tumor size, serous coat infiltration and lymphatic metastasis in colon cancer $(\mathrm{P}<0.05)$. In addition, the expression levels of $\mathrm{Pl} 3 \mathrm{~K}$ and pAkt were positively correlated with that of VEGF in colon cancer tissues $(\mathrm{P}<0.05)$. These results suggest that $\mathrm{PI} 3 \mathrm{~K} / \mathrm{Akt}$ signaling and VEGF signaling cross-talk with each other to promote the progression, metastasis and angiogenesis of colon cancer. In addition, our findings of the positive correlation between PI3K/Akt activation and VEGF expression in colorectal cancer tissues are largely consistent with the results of other studies that investigated the roles of PI3K/Akt and VEGF in other types of cancers, particularly epithelial cancers.

In conclusion, our data suggest that pAkt is an independent prognostic marker for colon cancer patients. Further large-scale and long-term follow-up studies may be useful in comfirming the prognostic value of pAkt for colon cancer.

\section{Acknowledgements}

This study was supported by a Province Key Lab grant (No. LS2010102) awarded by the Department of Education of Liaoning Province, China.

\section{References}

1. Yang L, Parkin DM, Li L, et al: Time trends in cancer mortality in China: 1987-1999. Int J Cancer 106: 771-783, 2003.

2. Duronio V: The life of a cell: apoptosis regulation by the PI3K/ PKB pathway. Biochem J 415: 333-344, 2008.

3. Hlobilkova A, Ehrmann J, Sedlakova E, Krejci V, Knizetova P, et al: Could changes in the regulation of the PI3K/PKB/Akt signaling pathway and cell cycle be involved in astrocytic tumor pathogenesis and progression? Neoplasma 54: 334-341, 2007.
4. Huang C, Huang R, Chang W, Jiang T, Huang K, et al: The expression and clinical significance of pSTAT3, VEGF and VEGF-C in pancreatic adenocarcinoma. Neoplasma 59: 52-61, 2012.

5. Kozlowski M, Naumnik W, Niklinski J, Milewski R, Dziegielewski P and Laudanski J: Vascular endothelial growth factor $\mathrm{C}$ and $\mathrm{D}$ expression correlates with lymph node metastasis and poor prognosis in patients with resected esophageal cancer. Neoplasma 58: 311-319, 2011

6. Wang F, Zhang P, Shi C, et al: Immunohistochemical detection of HSP27 and hnRNP K as prognostic and predictive biomarkers for colorectal cancer. Med Oncol: Aug 23, 2011 (Epub ahead of print).

7. Liang QL, Li ZY, Chen GQ, et al: Prognostic value of serum soluble Fas in patients with locally advanced unresectable rectal cancer receiving concurrent chemoradiotherapy. J Zhejiang Univ Sci B 11: 912-917, 2010.

8. Liang QL, Wang BR and Li GH: DcR3 and survivin are highly expressed in colorectal carcinoma and closely correlated to its clinicopathologic parameters. J Zhejiang Univ Sci B 10: 675-682, 2009.

9. Hasselblom S, Hansson U, Olsson M, Toren L, Bergstrom A, et al: High immunohistochemical expression of p-AKT predicts inferior survival in patients with diffuse large B-cell lymphoma treated with immunochemotherapy. Br J Haematol 149: 560-568, 2010.

10. Yoshioka A, Miyata H, Doki Y, Yasuda T, Yamasaki M, et al: The activation of Akt during preoperative chemotherapy for esophageal cancer correlates with poor prognosis. Oncol Rep 19: 1099-1107, 2008

11. Valkov A, Kilvaer TK, Sorbye SW, Donnem T, Smeland E, et al: The prognostic impact of Akt isoforms, PI3K and PTEN related to female steroid hormone receptors in soft tissue sarcomas. J Transl Med 9: 200, 2011.

12. Olsson AK, Dimberg A, Kreuger J and Claesson-Welsh L: VEGF receptor signalling - in control of vascular function. Nat Rev Mol Cell Biol 7: 359-371, 2006.

13. Dellinger MT and Brekken RA: Phosphorylation of Akt and ERK1/2 is required for VEGF-A/VEGFR2-induced proliferation and migration of lymphatic endothelium. PLoS One 6: e28947, 2011. 